EPJ Web of Conferences 41, 02001 (2013)

DOI: $10.1051 /$ epjconf/20134102001

(C) Owned by the authors, published by EDP Sciences, 2013

\title{
Enhancing temporal resolution in pump-probe experiments with noisy pulses
}

\author{
K. Meyer ${ }^{1}$, C. Ott ${ }^{1}$, P. Raith ${ }^{1}$, A. Kaldun ${ }^{1}$, Y. Jiang ${ }^{2}$, A. Senftleben ${ }^{1}$, M. Kurka ${ }^{1}$, R. Moshammer ${ }^{1}$, \\ J. Ullrich ${ }^{1,3}$, and T. Pfeifer ${ }^{1}$ \\ ${ }^{1}$ Max-Planck Institute for Nuclear Physics, Saupfercheckweg 1, 69117 Heidelberg, Germany \\ ${ }^{2}$ Shanghai Advanced Research Institute, Chinese Academy of Sciences, Pudong, Shanghai 201210, \\ China \\ ${ }^{3}$ Physikalisch-Technische Bundesanstalt (PTB), Bundesallee 100, 38116 Braunschweig, Germany
}

\begin{abstract}
We show how light fields that vary statistically in time (e.g. at FELs) enable higher temporal resolution than expected from their average pulse duration. An experimental example in deuterium molecules confirmed this general effect.
\end{abstract}

The coherence of laser light has led to important achievements in atomic, molecular and optical physics, from laser cooling to Bose-Einstein condensates, high-resolution spectroscopy and the generation of ultrashort laser pulses, to mention only a few. However, the recently developed Free-Electron Laser (FEL) sources exhibit statistically varying (i.e. mutually incoherent or only partially coherent) pulse shapes generated in the self-amplified stimulated emission (SASE) mode. Here, we present a novel concept to invert this apparent disadvantage and to enhance the temporal resolution in pump-probe experiments using noisy pulses [1]. Our method is demonstrated for an exemplary XUV-pump-XUV-probe experiment recently performed at the Free-Electron Laser at Hamburg (FLASH): the two-photon sequential double ionization (TPDI) of $\mathrm{D}_{2}$ [2]. The FEL pulses at an energy of $38 \mathrm{eV}$ were split up into pump and probe pulse and time delayed with respect to each other by a split mirror. In this experiment, the molecular wave-packet dynamics was measured by detecting the kinetic-energy release (KER) of the produced Coulomb-exploding $\mathrm{D}^{+}$-ions. An oscillatory motion of the nuclear wave packet at a period of $\sim 20$ fs was observed, coming by surprise as compared to the average pulse duration of $30 \mathrm{fs}$ of the FEL pulses.

The laser field experienced by the deuterium molecules is the sum of pump and probe field and thus the intensity depending on the time delay $\tau$ can be expressed by

$$
I_{\tau}(t)=|E(t)+E(t+\tau)|^{2} .
$$

The deuterium molecule first absorbs one photon at time $t^{\prime}$, leading to a singly ionized $\mathrm{D}_{2}{ }^{+}$ molecule. The absorption of a second photon at time $t^{\prime \prime}$ promotes the molecule into the Coulomb-exploding $\mathrm{D}_{2}^{2+}$ state. In order to model the ionization process we start from frequency-independent rate equations since the absorption of a photon always transfers the molecule into a continuum [cf. Figure 1(a)]. The number of doubly-ionized molecules can be determined by

This is an Open Access article distributed under the terms of the Creative Commons Attribution License 2.0, which permits unrestricted use, distribution, and reproduction in any medium, provided the original work is properly cited. 


$$
N_{\text {total }}(\tau) \propto \int_{-\infty}^{\infty} d t^{\prime \prime} \int_{-\infty}^{t^{\prime \prime}} d t^{\prime} I_{\tau}\left(t^{\prime}\right) I_{\tau}\left(t^{\prime \prime}\right)
$$

Replacing $t^{\prime}$ by the time difference $t_{c}=t^{\prime \prime}-t^{\prime}$ of the photon absorption and changing the integration order yields

$$
N_{\text {total }}(\tau) \propto \int_{0}^{\infty} d t_{c} \int_{-\infty}^{\infty} d t^{\prime \prime} I_{\tau}\left(t^{\prime \prime}-t_{c}\right) I_{\tau}\left(t^{\prime \prime}\right)=\int_{0}^{\infty} d t_{c} A_{c}^{(2)}\left(t_{c}, \tau\right) .
$$

$\mathrm{A}_{c}{ }^{(2)}\left(t_{c}, \tau\right)$ is the two-dimensional autocorrelation (2dAC) function where $t_{c}$, the time between the two photon absorption events, can be interpreted as the correlation time.
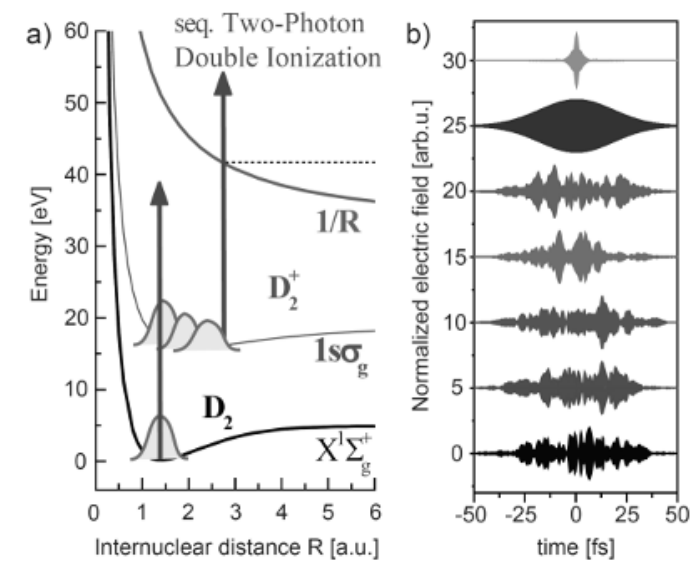

Fig. 1. Ingredients (matter and light) of the pump-probe experiment: (a) Potential curves of $\mathrm{D}_{2}$ and its cations. (b) Top to bottom: simulated temporal pulse shapes of the $1.12 \mathrm{fs}$ short pulse, the $30 \mathrm{fs}$ bandwidth-limited pulse and different FEL pulses.

In order to describe the total number of doubly-ionized deuterium molecules with a specific kinetic energy release (KER), we introduce a molecular response function $M\left(E_{K E R} t_{c}\right)$ mapping $t_{c}$ to $E_{K E R}$ by considering wave-packet propagation on the $1 \mathrm{~s} \sigma_{\mathrm{g}}$ state, leading to

$$
N_{\text {total }}\left(E_{K E R}, \tau\right) \propto \int_{0}^{\infty} d t_{c} M\left(E_{K E R}, t_{c}\right) A_{c}^{(2)}\left(t_{c}, \tau\right) .
$$

While the $2 \mathrm{dAC}$ function $\mathrm{A}_{c}{ }^{(2)}\left(t_{c}, \tau\right)$ provides information about the probe light structure (including its statistical fluctuations when averaging over several pulses), the molecular response function $\mathrm{M}\left(E_{K E R}, t_{c}\right)$ contains the physical information of the studied system. Both functions can even be considered generally applicable objects for sequential pump-probe schemes in other systems as well, where only the response function needs to reflect the system properties and the experimental observables. For the presented exemplary experiment it describes the evolution of the $\mathrm{D}_{2}{ }^{+}$wave packet in the $1 \mathrm{~s} \sigma_{\mathrm{g}}$ state. It is calculated by numerically solving the Schrödinger equation and Coulomb-explosion mapping KER $=1 / \mathrm{R}$. In our simulation, we generate a set of FEL pulses using the partial-coherence method [3]. The average pulse duration is taken into account by introducing a Gaussian time filter with a width of 30 fs. For comparison, our method is performed for a $1.12 \mathrm{fs}$ (FWHM) short pulse (which is the bandwidth limit of the measured average FEL spectrum) and a coherent (bandwidth limited) Gaussian pulse of 30 fs (FWHM) duration. The temporal shapes of the different pulses 
are shown in Figure 1(b). In Figure 2(a)-(c) the calculated 2dAC functions depending on the time delay $\tau$ between pump and probe pulse are displayed for the three different cases. For the 1.12 fs short pulse, three thin lines are observed, the origin of high temporal resolution, while for the $30 \mathrm{fs}$ bandwidth-limited pulse these lines become significantly broader, thus resulting in reduced temporal resolution. For the average of 2000 FEL pulses, a combination of the two aforementioned cases occurs: broad pedestals but featuring narrow lines along $\tau=t_{c}$, thus retaining high temporal resolution capability (cf. [1]). The resulting KER distributions depending on the time delay $\tau$ are shown in Figure 2(d)-(f). For the 1.12 fs short pulse, the wave-packet dynamics at a period of about $20 \mathrm{fs}$ is clearly resolved, while, by contrast, no dynamics can be seen using the $30 \mathrm{fs}$ bandwidth-limited pulse. In case of the FEL pulse average, the oscillatory dynamics is recovered and a closer analysis gives an oscillation period of $23 \pm 1 \mathrm{fs}$, in excellent agreement with the experiment [2].

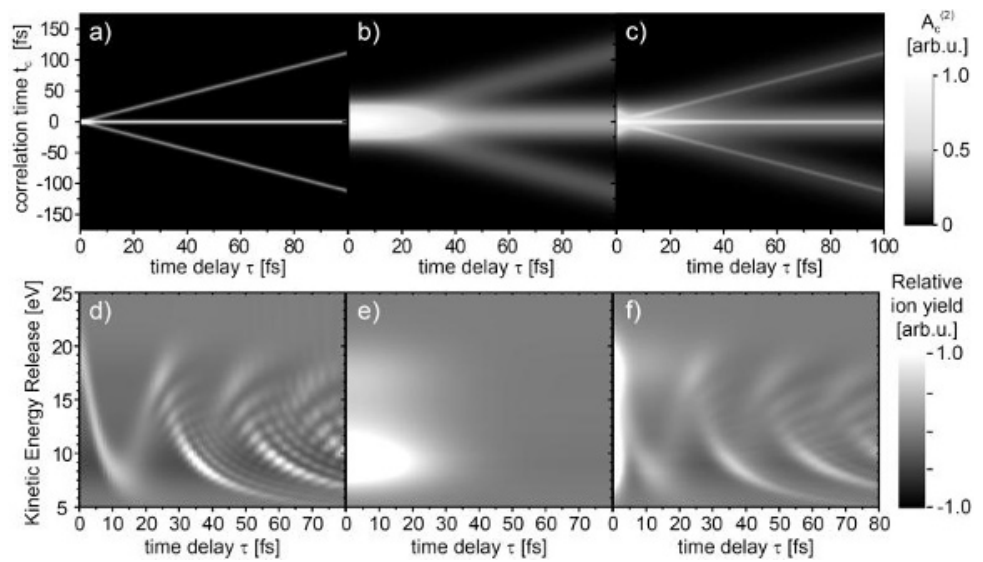

Fig. 2. Two-dimensional autocorrelation $(2 \mathrm{dAC})$ function $\mathrm{A}_{c}(2)$ and kinetic-energy release (KER). The $2 \mathrm{dAC}$ function dependent on the time delay $\tau$ is shown for a $1.12 \mathrm{fs}$ bandwidthlimited pulse (a), a 30 fs bandwidth-limited pulse (b) and an average of 2000 FEL pulses (c). The resulting KER-resolved fragment ion yield distributions for the $1.12 \mathrm{fs}$ bandwidth limited pulse (d), the $30 \mathrm{fs}$ bandwidth limited (e) and the averaged FEL pulses (f) demonstrate the resolution of molecular wave-packet dynamics far better $(\sim 2.6 \mathrm{fs})$ than the average pulse duration ( $30 \mathrm{fs}$ ) of the FEL pulses. The time-delay averaged yields at each KER are subtracted for the depicted relative ion yields.

We have shown that correlated-noise enables the measurement of dynamics on time scales that are shorter than the average pulse duration. Due to the universal validity of Eq. (4) where the response function can be adapted to various different target systems, this finding does not only have important consequences for FEL science as demonstrated here. It can lead to general paradigm shifts in ultrafast physics, including attosecond spectroscopy. For further details we refer to [1].

\section{References}

[1] K. Meyer et al., Phys. Rev. Lett. 108, 098302 (2012)

[2] Y. Jiang et al., Phys. Rev. A 81, 051402(R) (2010)

[3] T. Pfeifer, Y. Jiang, S. Düsterer, R. Moshammer, and J. Ullrich, Opt. Lett. 35, 3441 (2010) 